Cartas al Director

\title{
Metástasis coroideas y cáncer de pulmón
}

\section{Sr. Director:}

Recientemente se ha presentado en su revista el caso de un paciente de 65 años que presentó una alteración del campo visual, siendo finalmente diagnosticado de metástasis coroideas. Tras realizar el estudio pertinente, el enfermo fue diagnosticado de adenocarcinoma de pulmón. Se realizó tratamiento de quimioterapia, mejorando parcialmente la metástasis ocular, pero posteriormente se tuvo que realizar radioterapia, al presentar un desprendimiento de retina ${ }^{1}$.

En la discusión del caso el Dr. Villar Chamorro y colaboradores, comentan que únicamente se ha descrito un caso similar en la literatura; sin embargo hace algún tiempo se publicó en una revista española que está incluida en el índice médico español, un caso muy parecido ${ }^{2}$; y dado que no fue detectado en la búsqueda realizada por los autores del caso en la revista Oncología, nos parece interesante comentar brevemente algunos datos de interés.

Varón de 69 años, con antecedentes de consumo moderado de alcohol. Consultó al oftalmólogo al denotar pérdida progresiva de visión en el ojo derecho, de tres meses de evolución.

Se realizó un estudio con el oftalmoscopio, observándose la existencia de una tumoración en el borde de la hemiretina nasal del ojo derecho, compatible con metástasis coroideas, por lo que se remitió a la consulta de Oncología Médica.

En la anamnesis refería pérdida de 6 kilogramos de peso desde el principio del proceso. No refería ninguna otra sintomatología y en la exploración física solamente destacaba que se trataba de un paciente delgado.
Hemograma y bioquímica normales.

RNM de cráneo: metástasis intraocular afectando a la órbita derecha.

Radiografía de tórax: ligero engrosamiento pleural en el vértice del pulmón derecho.

TAC de tórax: En el lóbulo superior derecho se observó un pequeño nódulo espiculado con un tracto fibroso que le unía a la pleura y que parecía secuela de una tuberculosis antigua.

TAC de abdomen: normal.

Se realizó una PET para tratar de aclarar la naturaleza de la citada lesión, siendo el resultado compatible con lesión maligna en la localización anteriormente reseñada.

Se realizó en el Servicio de Radiología Intervensionista del Hospital Virgen de la Salud de Toledo una PAAF de la lesión, informándose de células malignas compatibles con adenocarcinoma moderadamente diferenciado.

Se efectúo radioterapia sobre el globo ocular afecto, iniciándose asimismo tratamiento de quimioterapia siguiendo el esquema carboplatino y paclitaxel.

Tras la radioterapia el paciente perdió completamente la visión del ojo.

Actualmente han pasado más de 12 meses desde el diagnóstico y el paciente se encuentra bien, sin datos de progresión de la enfermedad, siguiendo revisiones en la consulta de Oncología.

F. Marcos Sánchez, M. I. Albo Castaño, A. Viana Alonso, F. Juárez Ucelay

Servicio de Medicina Interna. Oncología Médica Hospital $N^{a} S^{a}$ del Prado Talavera de la Reina (Toledo) 


\section{Bibliografía}

1. Villar Chamorro E, Cobo Dols M, Gil Calle S, Montesa Pino A, Alcaide García J, Alés Díaz I, Gutiérrez Calderón V, Durán Ogalla G. Metástasis coroidea como debut de un carcinoma de pulmón no microcítico avanzado. Oncología 2006; 29(1): 43-46.
2. Marcos Sánchez F, Albo Castaño MI, Viana Alonso A, Franco Moreno AI, Gómez Soto FM, Juárez Ucelay F. Metástasis coroideas como primera manifestación del cáncer de pulmón. Investig Clin 2005; 8(3): 268-269.

Correspondencia:

Dr. F. Marcos Sánchez

Gregorio Corrochano, 1

E-45600 Talavera de la Reina (Toledo) 